\title{
Randomized Study Comparing Pre-Operative Glycemic Profile in Pediatric Cardiac Surgical Patients Administered Oral Carbohydrate Solution Preoperatively versus Those Kept Fasting
}

\author{
Uma Balasubramaniam1, Usha Kiran1, Suruchi Hasija1, V. Devagourou² \\ ${ }^{1}$ Department of Cardiothoracic and Vascular Anaesthesia, AIIMS, New Delhi, India \\ ${ }^{2}$ Department of Cardiothoracic and Vascular Surgery, AIIMS, New Delhi, India \\ Email: uma_dilli@yahoo.co.in
}

How to cite this paper: Balasubramaniam, U., Kiran, U., Hasija, S. and Devagourou, V. (2018) Randomized Study Comparing Pre-Operative Glycemic Profile in Pediatric Cardiac Surgical Patients Administered Oral Carbohydrate Solution Preoperatively versus Those Kept Fasting. World Journal of Cardiovascular Diseases, 8, 298-306. https://doi.org/10.4236/wjcd.2018.86029

Received: May 20, 2018

Accepted: June 11, 2018

Published: June 14, 2018

Copyright (ㅇ 2018 by authors and Scientific Research Publishing Inc. This work is licensed under the Creative Commons Attribution International License (CC BY 4.0).

http://creativecommons.org/licenses/by/4.0/

\begin{abstract}
Objectives: Hypoglycemia is a recognized danger in pediatric patients. Extended period of preoperative fasting in this subset of patients is not well tolerated with metabolic derangements. The oral carbohydrate loading preoperatively can ameliorate many adverse effects. The aim of this study was to compare the glycemic profile in pediatric cardiac surgical patients kept fasting preoperatively with those fed oral clear solutions of carbohydrate half hour prior to induction of anaesthesia. Also we tried to establish a correlation with other factors contributing to preoperative hypoglycemia. Methodology: We planned a randomized controlled study. Group A included patients who were kept fasting according to the ASA guidelines preoperatively and Group B included patients who received $2 \mathrm{ml}$ per $\mathrm{kg}$ of body weight of $10 \%$ Dextrose water as oral feeds half hour before the expected time of start of anaesthesia. Results: The mean (SD) preoperative BG concentrations were higher in group B $(102.5 \pm 16.97)$ as compared to group A $(64.08 \pm 25.37)$ ( $p$ value $<0.0001)$. Additionally in both the groups, cyanotics had a higher propensity towards decreased preoperative blood sugar levels (correlation coefficient -0.86 and -0.67 ) ( $p$ value $<0.05$ ). Conclusion: Preoperative oral carbohydrate preloading can develop as the easiest and cheapest path to better perioperative blood glucose concentration management in congenital cardiac disease children.
\end{abstract}

\section{Keywords}

Congenital Cardiac Disease, Hypoglycemia, Hypocalcemia, Oral Carbohydrate Solution, Preoperative, Cyanotics, Hematocrit 


\section{Introduction}

The perioperative period represents a crucial moment in the outcome of children with congenital cardiac disease (CCD). In this period, patients undergo significant changes in blood volume, plasma composition, dyselectrolytemia due to ongoing medications, and psychological perturbations, with important pathophysiological consequences [1]. The perpetuation of the pathological state can lead to occurrence of clinical-surgical complications [2]. Hypoglycemia is recognized as being a perioperative danger in paediatric practice predisposing to lethargy, irritability and metabolic acidosis finally culminating to complications like seizures. The brain can suffer irreversible injury during acute hypoglycemic episode [3]. During surgery, there is a rise in the plasma glucose level in normal adult, but it has been shown that children do not respond with a hyperglycemic reaction to the same degree.

In recent years, the pre-operative fasting for pediatric patients undergoing elective surgical procedures has seen changing trends towards shorter time intervals. Fasting for many hours prior to anaesthesia can lead to release of stress hormones and pro-inflammatory cytokines leading to insulin resistance, hyperglycemia and catabolism, thereof increasing percentages of postoperative complications [4]. The preoperative administration of carbohydrates has beneficial effects on the metabolic response to stress, mainly by decreasing tendency to hypoglycemia, decreasing catabolism and promoting earlier postoperative recovery times [5]. The prescription of these liquids helps preserve intravascular volume, improving hemodynamics and facilitating obtaining peripheral venous access. Shortening the preoperative fasting contributes to reducing hunger, thirst and anxiety of patients, and so they show greater cooperation at the time of anesthesia induction. The current ASA fasting guidelines are commonly applied to all pediatric patients irrespective of the nature of disease, surgery planned and demographic variables [6].

A cardiac anaesthesiologist must carefully consider the pathophysiology of the CCD in the child, the nil per os (NPO) status and patient-specific methods of treatment to optimally manage the preoperative glycemic status. In the population of pediatric cardiac surgical patients, there is no study evaluating the glycemic status pre-operatively and generalized fasting guidelines have been applied to cyanotics as well as acyanotics. Also, no study has evaluated effect of preoperative administration of carbohydrates in this group of patients.

We hence, conducted a randomized controlled study (CTRI number: CTRI/2018/05/014033) among pediatric patients with congenital cardiac disease to investigate the events of hypoglycemia pre operatively and associated complications in those kept fasting according to ASA guidelines as compared to those administered preoperative oral carbohydrate solution.

\section{Methods}

After approval by the institutional ethics committee and written informed con- 
sent, 120 patients aged between one day to 5 years belonging to ASA grade II III undergoing elective cardiac surgery for congenital cardiac disease in the year 2015 and 2016 were enrolled in this randomized controlled study. The patients were allocated into two groups based on sealed envelope technique. Group A included patients who were kept fasting according to ASA guidelines preoperatively and Group B included patients who received $2 \mathrm{ml}$ per $\mathrm{kg}$ of body weight of $10 \%$ Dextrose water as oral feeds half hour before the expected time of start of anaesthesia. Patients on prior ventilatory support, corticosteroid therapy, diabetes, sepsis and metabolic disorders were excluded from the study.

All patients underwent preanaesthetic examination one day prior to surgery. Premedication was given with syrup Promethazine at a dose of $1 \mathrm{mg} / \mathrm{kg}$. Inside the OR standard monitoring i.e. electrocardiography, pulse oximetry, non-invasive blood pressure was instituted. Intravenous access was secured and immediately blood glucose concentrations were measured from the venous blood which returned to the hub of the catheter inserted. Blood glucose analysis was done using glucometer.

Hypoglycemia was defined by standard definitions:

Neonatal hypoglycemia-blood glucose level of less than $30 \mathrm{mg} / \mathrm{dL}(1.65$ $\mathrm{mmol} / \mathrm{L})$ in the first 24 hours of life and less than $45 \mathrm{mg} / \mathrm{dL}(2.5 \mathrm{mmol} / \mathrm{L})$ thereafter till one year of age [7].

Hypoglycemia-blood glucoselevels less than $60 \mathrm{mg} / \mathrm{dl}(3.3 \mathrm{mmol} / \mathrm{l})$ till 2 years of life and less than $70 \mathrm{mg} / \mathrm{dl}(3.9 \mathrm{mmol} / \mathrm{l})$ thereafter [8] [9].

Hypoglycemia, if diagnosed, was corrected with $10 \%$ dextrose containing solutions @ $2.5 \mathrm{ml} / \mathrm{kg}$ intravenously. Thereafter, anaesthesia was conducted as per routine protocol. Arterial line and central venous catheter were secured under all aseptic precautions after induction of anaesthesia. Arterial blood gas analysis was done immediately after this to obtain baseline hematocrit and calcium levels in addition to other parameters.

Statistical analysis: The primary observation of the study was blood glucose concentrations prior to induction of anaesthesia. Secondary observations were hours of pre-operative fasting, signs of pre-operative hypoglycemia like sweating, lethargy, excessive crying, baseline calcium and hematocrit levels and any complications like seizures, aspiration, hemodynamic instability etc. Based on the results of a previous study, the blood glucose concentration in children administered oral carbohydrate solution preoperatively was $82.9 \mathrm{mg} / \mathrm{dl}$ (SD 6.02 $\mathrm{mg} / \mathrm{dl})$. To detect a difference of $10 \%$ for the same, prospective power analysis at $90 \%$ power and a 0.05 level of significance ( 2 sided), a sample size of 60 patients in each group was required. Therefore, we studied a total of 120 patients. We used SAS $9.0^{\mathrm{TM}}$ statistical software (SAS Inc., Cary, North Carolina, USA). Data were tested for normality using Kolmogorov-Smirnov test. Student's t-test was used to analyze the parametric variables. Categorical measures were compared using chi-square test. Pearson's correlation coefficient test was used to find relation between the congenital condition (cyanotic vs. acyanotic) and preoperative 
blood glucoseconcentrations, baseline calcium levels and hematocrit. The value of correlation coefficient was calculated between -1 and +1 . Linear regression was used to determine relationship of preoperative blood glucose concentration with independent variables. A $p$-value of $<0.05$ was considered statistically significant.

\section{Results}

There were 120 patients enrolled in the study with no dropouts. Patients in both the groups had comparable demographic variables like age, gender, weight, ASA, cardiac condition (Table 1).

The primary outcome of the study was to compare the preoperative blood glucose concentrations between groups $\mathrm{A}$ and $\mathrm{B}$. The mean (SD) preoperative blood glucose concentrations were higher in group B (102.5 \pm 16.97$)$ as compared to group A $(64.08 \pm 25.37)$. The difference was statistically significant with a $p$ value $<0.0001$.

Also within each group the cardiac condition i.e. cyanotics vs acyanotics was correlated with the blood glucose concentrations. There was a strong negative correlation $(-0.86)$ of the condition with the blood glucose levels in group A. The correlation was moderately negative in group B $(-0.67)$. Thus in both the groups, cyanotics had a higher propensity towards decreased preoperative blood sugar levels with a $p$ value $<0.05$.

Additionally we compared the preoperative ionic calcium levels as well as hematocrit in both the groups. They were comparable with $\mathrm{p}$ values of 0.14 and 0.87 respectively.

From our data correlation, we also found that irrespective of the blood glucoseconcentration and fasting status, the calcium levels were strongly negatively correlated $(-0.78)$ with the cardiac condition i.e. children with cyanotic congenital heart disease had decreased baseline calcium levels (Figure 1).

Using linear regression analysis, we found that gender, cardiac condition and the study group to be independent factors in determining the preoperative blood glucose concentrations $(p<0.05)$. Based on this, the linear regression model for pre operative blood glucose levels was represented as:

Preoperative BS $=88.10+39.63 \times($ Group B $)-6.42($ Female $)-35.22$ (Cyanotic)

No complication was recorded in either group during the study period. Thirty nine (65\%) patients in group A had signs of hypoglycemia like excessive crying, increased sweating and irritability in the preoperative period which was either reported by the parent or the attending nurse. Of these twenty eight patients were with cyanotic heart disease. Only fourteen patients (23\%) in group B had similar symptoms and all of them had cyanotic heart disease.

The total duration of fasting (time from last clear fluid taken) was comparable in both the groups with a mean (SD) duration of fasting in group A of 364.06 (193.51) minutes and in group B of 311.84 (185.84) minutes with a $\mathrm{p}$ value of 0.13 . 
Table 1. Characteristics of patients in group A and B. Values are mean (SD) or number (proportion).

\begin{tabular}{cccc}
\hline Demographic variables & $\begin{array}{c}\text { Group A } \\
\mathbf{n}=\mathbf{6 0}\end{array}$ & $\begin{array}{c}\text { Group B } \\
\mathbf{n}=60\end{array}$ & $\boldsymbol{p}$ value \\
Age; years & $3.15(3.58)$ & $2.56(3.01)$ & 0.33 \\
Gender; M/F & $42 / 18$ & $41 / 19$ & 0.64 \\
Weight; kg & $13.14(8.45)$ & $11.9(8.22)$ & 0.41 \\
Cardiac condition; cyanotic/acyanotic & $37 / 23$ & $39 / 21$ & 0.70 \\
$\begin{array}{c}\text { Preoperative blood glucose } \\
\text { concentrations (mg/dl); mean (SD) }\end{array}$ & $64.08(25.37)$ & $102.5(16.97)$ & $<0.0001$ \\
\hline
\end{tabular}

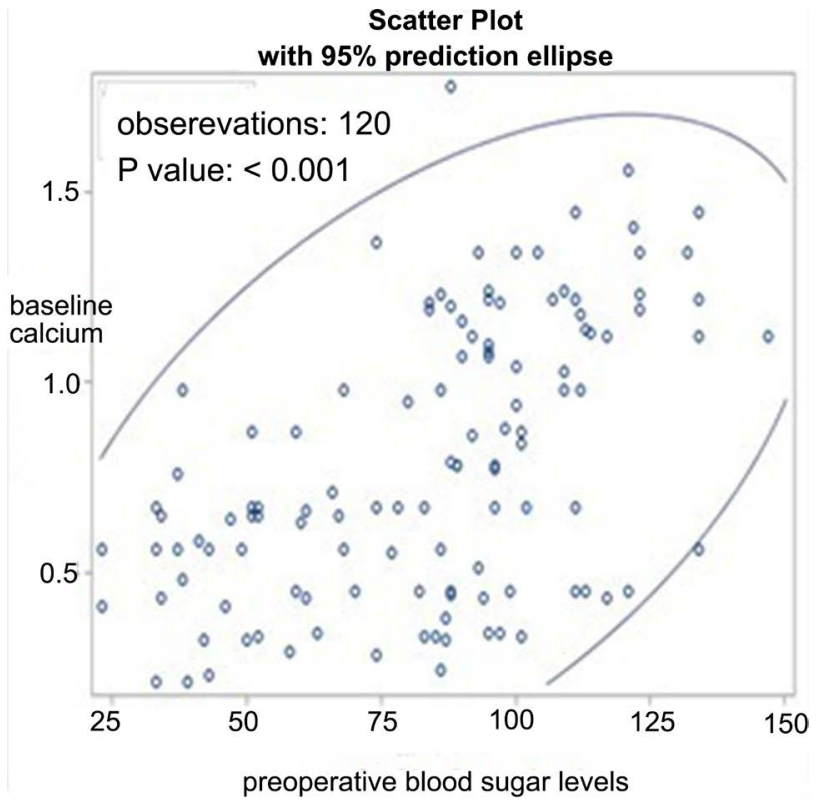

Figure 1. Scatter plot diagram correlating preoperative blood glucose levels with baseline calcium levels.

\section{Discussion}

"Nil per oral" has been the dictum in perioperative anaesthesia care. This approach has recently been extensively debated worldwide within the anaesthesia fraternity. Studies in adults found that the use of drinks containing carbohydrates in the preoperative period determines greater satisfaction, less irritability, lower incidence of vomiting, better gastric emptying and smaller organic response to surgical stress [10]. In children it has also been observed that the use of carbohydrate drinks before the surgical procedure is safe [11]. In our study, we studied pediatric patients with congenital heart disease to observe there response to carbohydrate rich oral fluids in the preoperative period. Patients administered such carbohydrate solution had lower incidence of hypoglycemia with mean blood glucose levels of $102.5 \mathrm{mg} / \mathrm{dl}$ as compared to those kept fasting as per protocol $(64.08 \mathrm{mg} / \mathrm{dl})$.

The American Society of Anesthesiologists recommends for patients of any 
age, previously healthy and who will undergo elective surgical procedures with the use of anesthesia, fasting periods of 2 hours to clear liquids (water, juices without pulp, carbohydrate drinks, teas); 4 hours for breast milk; 6 hours for formula milk, non-human milk and snacks (toast and clear liquids); and 8 hours to fatty foods or fried food [6]. However, these fasting protocols become difficult to be enforced due to sleeping pattern of the child, parental anxiety, varying institutional practices, expected and actual time of shifting to OR etc. [12]. Hence children may remain fasting for longer periods than recommended, sometimes culminating to more than 12 hours. These long periods of food abstention can lead to thirst and dehydration, hunger, hypoglycemia (especially children who have lower reserves of glycogen), irritability, headache and non-cooperative and inconsolable child at induction of anaesthesia. Also they are liable to delayed arousal from anesthesia, causing unpleasant experience for these small patients [13] [14]. In our study we experienced that patients were fasting for more than recommended hours in both the groups despite clear instructions to parents. Most of these were due to the child going off to sleep before taking there last meal. The second common reason was due to delay in surgery as they were posted as second or third case in the operative list.

Statistical analysis within each group demonstrated intra-group variability with cyanotics being at higher risk for hypoglycemia as compared to acyanotics in both the groups. This negative correlation suggests that fasting guidelines cannot be standardized to all pediatric patients with congenital heart disease. Also intravenous fluid started for cyanotic patients in the preoperative period cannot become a substitute for oral preloading. Compared to intravenous supply of glucose, preoperative oral supply of carbohydrate solution decreases the gastric residual volume [15].

The one area of concern with such an oral carbohydrate preloading is the risk of pulmonary aspiration due to larger residual gastric volume. No child in either group had any regurgitation or aspiration against that expected with giving oral fluids 30 minutes prior to shifting to OR. Gastric emptying for liquids in children is fast. Schmitdz et al observed that the average gastric emptying time as seen by serial MRI imaging in 16 children between 6 - 14 years who were offered volume of $7 \mathrm{ml} / \mathrm{kg}$, was only 30 minutes [16]. Schmidt et al. found no difference in residual volume or gastric $\mathrm{pH}$ in children after 1 - 2 hours of clear fluid intake [17]. According to Senayli et al., the use of the ready-to-use solution of carbohydrates in children is safe and does not alter $\mathrm{pH}$ of gastric contents compared to children fasting until surgery and receiving drinking water [18].

Preoperative thirst has been suggested to be the main contributory factor of patient discomfort, followed by hunger and anxiety [19]. Clear drinks alleviate thirst but their effect on hunger is inconclusive [20] [21]. Children administered oral carbohydrate preload in our study had less evidence of discomfort, irritability, dry mouth, excessive crying etc.

The cyanotic patients in both the groups had lower levels of ionic calcium as 
compared to acyanotics. This finding can be attributed to the fact that majority of congenital heart disease have a genetic contributing factor. Commonly most of these patients have been reported to have abnormality in chromosome $22 \mathrm{q}$ which is linked to various genetic disorders like CATCH22, Digeorge syndrome etc [22] [23] [24]. A low level of calcium is contemplated in these conditions [25] [26]. We did not perform genetic screening in our patients to rule out Digeorge syndrome. Cyanotics can be evaluated for Digeorge syndrome with low calcium levels serving as an indirect indicator.

Our study had a few limitations. We did not assess the postoperative glycemic response of our patients. We could not limit our study to only those taken as first case in the morning of surgery. Hence it produced wide variability in fasting times. We have used a very small volume of oral preload as compared to other studies. That could be the contributing factor to no complication of aspiration.

Oral preload with carbohydrate rich solution has been successfully imple-mented in many surgical fields with improved outcomes. There advantages in cardiac surgical patients need further introspection especially in the pediatric group to avoid the unpleasant long duration of fasting. Also no study has defined an ideal preloading oral carbohydrate drink. Our study highlights key issues of tolerance, compliance and safety of preoperative oral supply of carbohydrate preparations in children. The present time demands a need to revisit fasting guidelines and base it on type of patient profile rather than generalizing it for all.

\section{Conclusion}

Preoperative oral carbohydrate preloading can develop as the easiest and cheapest path to better perioperative blood glucose concentration management in congenital cardiac disease children. This can reduce the hypoglycemia associated adverse events and improve cooperation during preoperative period. From the observations in our study we highlight the need to individualise fasting protocols based on the particular condition being addressed.

\section{References}

[1] Greeley, W.J., Steven, J.M., Nicolson, S.C. and Kern, F.H. (2000) Anesthesia for Pediatric Cardiac Surgery. In: Miller, R.D., Ed., Anesthesia, Churchill Livingstone, Philadelphia, 1805-1847.

[2] Ljungqvist, O., Nygren, J., Soop, M., et al. (2005) Metabolic Perioperative Management: Novel Concepts. Current Opinion in Critical Care, 11, 295-299. https://doi.org/10.1097/01.ccx.0000166395.65764.71

[3] Nasraway Jr., S.A. (2007) Sitting on the Horns of a Dilemma: Avoiding Severe Hypoglycemia while Practicing Tight Glycemic Control. Critical Care Medicine, 35, 2435-2437. https://doi.org/10.1097/01.CCM.0000286520.50104.51

[4] Li, L., Wang, Z., Ying, X., et al. (2012) Preoperative Carbohydrate Loading for Elective Surgery: A Systematic Review and Meta-Analysis. Surgery Today, 42, 613-624. https://doi.org/10.1007/s00595-012-0188-7 
[5] Noblett, S.E., Watson, D.S., Huong, H., et al. (2006) Pre-Operative Oral Carbohydrate Loading in Colorectal Surgery: A Randomized Controlled Trial. Colorectal Disease, 8, 563-569. https://doi.org/10.1111/j.1463-1318.2006.00965.x

[6] (2017) Practice Guidelines for Preoperative Fasting and the Use of Pharmacologic Agents to Reduce the Risk of Pulmonary Aspiration: Application to Healthy Patients Undergoing Elective Procedures: An Updated Report by the American Society of Anesthesiologists Task Force on Preoperative Fasting and the Use of Pharmacologic Agents to Reduce the Risk of Pulmonary Aspiration. Anesthesiology, 126, 376-393. https://doi.org/10.1097/ALN.0000000000001452

[7] Srinivasan, G., Pildes, R.S., Cattamanchi, G., et al. (1986) Plasma Glucose Values in Normal Neonates: a New Look. Journal of Pediatrics, 109, 114-117. https://doi.org/10.1016/S0022-3476(86)80588-1

[8] Brown, T.C., Connelly, J.F., Dunlop, M.E., et al. (1980) Fasting Plasma Glucose in Children. Australian Paediatric Journal, 16, 28-29. https://doi.org/10.1111/j.1440-1754.1980.tb02480.x

[9] Pagana, K.D. and Pagana, T.J. (2003) Mosby’s Diagnostic and Laboratory Test Reference. 6th Edition, Mosby, Elsevier Science, Philadelphia.

[10] Oliveira, K.G., Balsan, M., Oliveira Sde, S., et al. (2009) Does Abbreviation of Preoperative Fasting to Two Hours with Carbohydrates Increase the Anesthetic Risk. Revista Brasileira de Anestesiologia, 59, 577-584. https://doi.org/10.1016/S0034-7094(09)70082-0

[11] Andersson, H., Zarén, B. and Frykholm, P. (2015) Low Incidence of Pulmonary Aspiration in Children Allowed Intake of Clear Fluids until Called to the Operating Suite. Paediatric Anaesthesia, 25, 770-777. https://doi.org/10.1111/pan.12667

[12] Moyao-García, D., Corrales-Fernández, M.A., Blanco-Rodríguez, G., SánchezHernández, E. and Nava-Ocampo, A.A. (2001) Benefits of Oral Administration of an Electrolyte Solution Interrupting a Prolonged Preoperatory Fasting Period in Pediatric Patients. Journal of Pediatric Surgery, 36, 457-459. https://doi.org/10.1053/jpsu.2001.21614

[13] Castillo-Zamora, C., Castillo-Peralta, L.A. and Nava-Ocampo, A.A. (2005) Randomized Trial Comparing Overnight Preoperative Fasting Period vs Oral Administration of Apple Juice at 06:00-06:30 am in Pediatric Orthopedic Surgical Patients. Paediatric Anaesthesia, 15, 638-642. https://doi.org/10.1111/j.1460-9592.2005.01517.x

[14] Gebremedhn, E.G. and Nagaratnam, V.B. (2014) Audit on Preoperative Fasting of Elective Surgical Patients in an African Academic Medical Center. World Journal of Surgery, 38, 2200-2204. https://doi.org/10.1007/s00268-014-2582-3

[15] Kaska, M., Grosmanova, T., Havel, E., et al. (2010) The Impact and Safety of Preoperative Oral or Intravenous Carbohydrate Administration versus Fasting in Colorectal Surgery-A Randomized Controlled Trial. Wiener klinische Wochenschrift, 122, 23-30. https://doi.org/10.1007/s00508-009-1291-7

[16] Schmitz, A., Kellenberger, C.J., Liamlahi, R., et al. (2011) Gastric Emptying after Overnight Fasting and Clear Fluid Intake: A Prospective Investigation Using Serial Magnetic Resonance Imaging in Healthy Children. British Journal of Anaesthesia, 107, 425-429. https://doi.org/10.1093/bja/aer167

[17] Schmidt, A.R., Buehler, P., Seglias, L., et al. (2015) Gastric pH and Residual Volume after 1 and $2 \mathrm{~h}$ Fasting Time for Clear Fluids in Children. British Journal of Anaesthesia, 114, 477-482. https://doi.org/10.1093/bja/aeu399

[18] Senayli, Y., Senayli, A., Erkorkmaz, U., et al. (2006) Effects of the Preoperative Nu- 
tritional Beverage, Water and Fasting Policies on the Gastric Acidity Levels during Surgery: A Clinical Pilot Study at the Pediatric Age Group. The Internet Journal of Anesthesiology, 14.

[19] Madsen, M., Brosnan, J. and Nagy, V.T. (1998) Perioperative Thirst: A Patient Perspective. Journal of PeriAnesthesia Nursing, 13, 225-228. https://doi.org/10.1016/S1089-9472(98)80018-X

[20] Splinter, W.M., Stewart, J.A. and Muir, J.G. (1990) Large Volumes of Apple Juice Preoperatively Do Not Affect Gastric $\mathrm{pH}$ and Volume in Children. Canadian Journal of Anesthesia, 37, 36-39. https://doi.org/10.1007/BF03007481

[21] Splinter, W.M., Stewart, J.A. and Muir, J.G. (1989) The Effect of Preoperative Apple Juice on Gastric Contents, Thirst, and Hunger in Children. Canadian Journal of Anesthesia, 36, 55-58. https://doi.org/10.1007/BF03010888

[22] Markert, M.L., Sarzotti, M. and Ozaki, D.A. (2003) Thymus Transplantation in Complete DiGeorge Syndrome: Immunologic and Safety Evaluations in 12 Patients. Blood, 102, 1121-1130. https://doi.org/10.1182/blood-2002-08-2545

[23] Soares, G., Alvares, S., Rocha, C., et al. (2005) Congenital Heart Defects and Chromosomal Anomalies Including 22q11 Microdeletion (CATCH 22). Revista Portuguesa de Cardiologia, 24, 349-371.

[24] Marino, B., Digilio, M.C., Toscano, A., et al. (2001) Anatomic Patterns of Conotruncal Defects Associated with Deletion 22q11. Genetics in Medicine, 3, 45-48. https://doi.org/10.1097/00125817-200101000-00010

[25] Kitsiou-Tzeli, S., Kolialexi, A., Fryssira, H., et al. (2004) Detection of 22q11.2 Deletion among 139 Patients with DiGeorge/Velocardiofacial Syndrome Features. In Vivo, 18, 603-608.

[26] Bassett, A.S., Chow, E.W., Husted, J., et al. (2005) Clinical Features of 78 Adults with 22q11 Deletion Syndrome. American Journal of Medical Genetics Part A, 138, 307-313. https://doi.org/10.1002/ajmg.a.30984 